\title{
Associations among Water, Sanitation, and Hygiene, and Food Exposures and Typhoid Fever in Case-Control Studies: A Systematic Review and Meta-Analysis
}

\author{
Sarah Brockett, ${ }^{1}$ Marlene K. Wolfe, ${ }^{2,3 *}$ Asa Hamot, ${ }^{2}$ Grace D. Appiah, ${ }^{4}$ Eric D. Mintz, ${ }^{4}$ and Daniele Lantagne ${ }^{2}$ \\ ${ }^{1}$ Tufts University School of Medicine, Boston, Massachusetts; ${ }^{2}$ Department of Civil and Environmental Engineering, Tufts University, Medford, \\ Massachusetts; ${ }^{3}$ Department of Civil and Environmental Engineering, Stanford University, Stanford, California; ${ }^{4}$ Centers for Disease Control and \\ Prevention, Atlanta, Georgia
}

\begin{abstract}
Typhoid fever transmission occurs through ingestion of food or water contaminated with Salmonella Typhi, and case-control studies are often conducted to identify outbreak sources and transmission vehicles. However, there is no current summary of the associations among water, sanitation, and hygiene (WASH); and food exposures and typhoid from case-control studies. We conducted a systematic review and meta-analysis of case-control studies to evaluate the associations among typhoid fever and predicted WASH or food exposure risk factors (13), and protective factors (7). Overall, 19 manuscripts describing 22 case-control studies were included. Two studies were characterized as having low risk of bias, one as medium risk, and 19 as high risk. In total, nine of 13 predicted risk factors were associated with increased odds of typhoid (odds ratio $[\mathrm{OR}]=1.4-2.4, I^{2}=30.5-74.8 \%$.), whereas five of seven predicted protective factors were associated with lower odds of typhoid $\left(\mathrm{OR}=0.52-0.73, I^{2}=38.7-84.3 \%\right)$. In five types of sensitivity analyses, two $(8 \%)$ of 26 summary associations changed significance from the original analysis. Results highlight the following: the importance of household hygiene transmission pathways, the need for further research around appropriate food interventions and the risk of consuming specific foods and beverages outside the home, and the absence of any observed association between sanitation exposures and typhoid fever. We recommend that typhoid interventions focus on interrupting household transmission routes and that future studies provide more detailed information about WASH and food exposures to inform better targeted interventions.
\end{abstract}

\section{INTRODUCTION}

Typhoid fever is an acute febrile illness caused by Salmonella enterica serovar Typhi (Typhi). ${ }^{1}$ Humans are the only host and reservoir for Typhi, and transmission occurs through the ingestion of fecally contaminated food or water. ${ }^{2}$ Please note that, as per the CDC and WHO guidance, we have chosen to use the nomenclature "Typhi" alone to describe Typhi without a species name in this article.

Most typhoid fever cases occur in endemic areas of lowand middle-income countries (LMIC), in populations without access to safe water, sanitation, and hygiene (WASH) or safe food-handling practices. ${ }^{3}$ The estimated global burden of typhoid fever is 10.9 million cases and 116,800 deaths annually, most of which are in children. ${ }^{3-5}$ Several factors make control of typhoid fever challenging: including its nonspecific clinical presentation, difficult laboratory diagnosis, increasing antimicrobial resistance, and the persistent and asymptomatic shedding of bacteria by convalescent and chronic asymptomatic carriers.

Symptoms of typhoid fever vary and include low-grade fever, malaise, dry cough, diarrhea, constipation, and abdominal pain. Symptoms typically last 3-4 weeks if untreated, although complications can develop, including gastrointestinal bleeding, intestinal perforation, and typhoid encephalopathy. ${ }^{1,6}$ The gold standard for typhoid diagnosis is isolation and identification of Typhi from culture of blood, stool, or bone marrow; however, healthcare facilities in LMIC often lack capacity for performing bacterial culture. Thus, many typhoid cases are diagnosed clinically or with unreliable serologic tests, whereas others remain undiagnosed and inappropriately treated. ${ }^{7}$ Following infection, patients may shed Typhi in their stool or urine

* Address correspondence to Marlene K. Wolfe, Department of Civil \& Environmental Engineering, Stanford University, 473 Via Ortega, Stanford, CA 94305. E-mail: marlene.wolfe@stanford.edu for 1-12 months, and up to $5 \%$ of patients will become asymptomatic chronic carriers, potentially excreting the bacteria for many years. ${ }^{1}$

Given the fecal-oral transmission route and persistent shedding by asymptomatic carriers in endemic populations, $\mathrm{WASH}$, and food-handling interventions are critical control measures to interrupt typhoid transmission. ${ }^{1}$ Water interventions improve the quality, quantity, or management of water. Sanitation interventions separate feces from human contact. Hygiene interventions refer to the conditions and practices that remove pathogens from the surfaces of an individual (handwashing, bathing, etc.) or the home environment (cleaning or disinfection). ${ }^{8-10}$ Food-handling interventions (food interventions) refer to the safe production, processing, and storage of foods, with a focus on high-risk foods commonly associated with transmission risk. ${ }^{1,11}$

Sources of typhoid infection, risk factors for transmission, and protective factors are investigated so that targeted interventions can be implemented. Case-control studies, which are relatively quick and inexpensive, are often used during outbreak investigations and in endemic settings. ${ }^{11}$ Although unable to determine causality, case-control studies can indicate specific protective or harmful exposures and practices, and facilitate retrospective comparisons of these exposures and practices across outbreaks. By identifying the most commonly identified WASH and food exposures and practices associated with typhoid acquisition and protection, reviews of case-control studies can suggest specific common intervention targets.

One or more WASH and food interventions are usually implemented during typhoid outbreaks, and these interventions may also confer protection against other faeco-orally transmitted diseases. However, little is known about which risky and protective WASH and food exposures are most important for typhoid in particular. ${ }^{12}$ To our knowledge, no systematic analysis of the evidence on WASH and food 
exposures and practices from case-control studies of typhoid fever has been published. To fill this evidence gap, we performed a systematic review of typhoid case-control studies to summarize the associations among WASH and food exposures and typhoid risk.

\section{METHODS}

We conducted a systematic review of the published literature to evaluate the association among WASH and food exposures and typhoid risk. The review was developed based on the guidelines for the Preferred Reporting Items for Systematic Reviews and Meta-Analyses (PRISMA) ${ }^{12}$ and included the development of the following: 1) definitions of risk and protection, 2) a search strategy, 3) inclusion criteria, 4) a selection and data extraction strategy, 5) framework for appraising the risk of bias, and 6) an analysis plan. The protocol was registered on the PROSPERO website before screening. ${ }^{13}$

Theory of risk and protection. Six groups of exposures that could theoretically have an impact on typhoid transmission were defined a priori: five WASH exposure groups that included water source, water treatment, water management, sanitation, and hygiene, and food exposures including the type of food and food practices. These exposures focus on pathways that represent individual-level risk factors, rather than the introduction of Typhi into community-level water or food supply. Although addressing the upstream introduction of typhoid into a community is critical to eliminate the disease, most case-control studies focused on more proximate risk factors related to transmission, and so this review focuses on preventing transmission after upstream exposure. During data extraction, all relevant exposures detailed in included casecontrol studies were categorized into one of these groups based on the presentation in the selected articles and in accordance with the UNICEF/WHO Joint Monitoring Program (JMP) definitions when possible. Because these groups were defined during analysis, their definitions are further described with results. During analysis, the exposures included within each WASH group were subdivided into predicted protective factors and predicted risk factors based on the same UNICEF/ WHO JMP definitions, and whether the exposure provided a barrier between feces and humans as detailed in the F-diagram (Figure 1). ${ }^{9,10,14}$

Categorization of food exposures was based on a description of either a food management practice or a specific

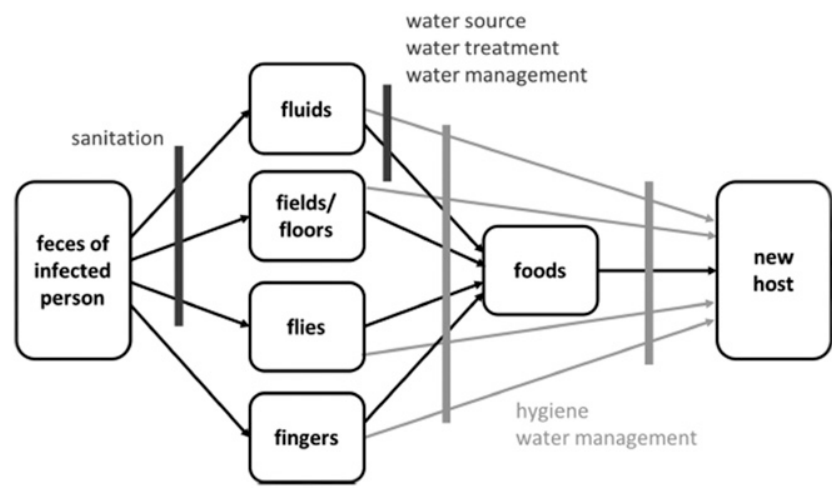

FIGURE 1. F-diagram showing pathways of fecal-oral disease and opportunities to interrupt transmission. type of food. Based on descriptions in the included articles, the food exposure group was subdivided into three categories: 1) food management practices, 2) the location of meals, and 3) the consumption of a specific food. Food practices were subcategorized into predicted protective factors and predicted risk factors based on the Safer Food guidelines developed by the WHO. ${ }^{12}$ Because there were few studies describing each of these behaviors, we combined the presence or absence of behaviors, including cooking food thoroughly and reheating cooked food, keeping food at safe temperatures through refrigeration, and using hygienic practices in food preparation and consumption into these two exposure categories. The location of meals was subcategorized as within or outside the home. Consumption of a specific food was subcategorized into food categories (e.g., vegetables and fruits) depending on types of food found during the analysis.

Exposures were chosen for inclusion based on the presence of sufficient data among the selected studies. To allow for sufficient data for meta-analysis, results are only reported for predicted protective or risky WASH and food factors with at least five reported exposures from case-control studies in at least three included articles.

Search strategy. In June 2018, the databases Web of Science (WOS), Medline (Pubmed), and Embase were searched using the following string: (["case control" or "case control stud" or "retrospective"] and "typhoid"). Because WASH and food exposures are often evaluated but not highlighted in the abstracts of these articles, the search was kept intentionally broad without reference to WASH or food exposures. The search was limited to peer-reviewed articles published between January 1, 1990 and June 7, 2018 in English. References were stored in EndNote (Boston, MA), and duplicates were deleted.

Inclusion criteria. Inclusion criteria were defined a priori according to the populations, interventions, comparisons, outcomes, and study types (PICOS) framework, a model recommended by the Cochrane Library to structure rigorous reviews on health-related questions. ${ }^{15}$

Populations. Eligible studies included populations affected by typhoid with all ages, genders, and socioeconomic statuses included. As most typhoid-endemic areas and typhoid outbreaks occur in LMIC, populations in countries classified as high income or upper-middle income by the World Bank list of economies were excluded. ${ }^{16}$

Interventions/exposures. Eligible studies were defined as including evaluations of eligible WASH or food exposures, rather than interventions. Using this definition, protective exposures represent interventions that should limit typhoid transmission, whereas risky exposures represent a gap in prevention that may allow for typhoid transmission. Studies were excluded if they were designed to specifically evaluate a typhoid vaccination program as the relationships between typhoid vaccinations and WASH or food exposures have not been studied thoroughly. ${ }^{16}$

Comparisons. Because we evaluated exposures rather than interventions, comparisons of treatment conditions were not relevant for inclusion.

Outcomes. Eligible studies reported an association between typhoid and at least one WASH or food exposure using an odds ratio (OR).

Study types. Only case-control studies were eligible for review. 
Selection and data extraction. Studies identified based on the aforementioned search criteria were selected for inclusion based on a two-phase screening process by two independent authors at each stage, including data extraction. First, a title and abstract screening was used to exclude studies in which the outcome was not typhoid cases, the study design was not case-control, or the target population was a high- or upper middle-income country. Then, the full text of remaining articles was examined, and studies that did not meet the PICOS criteria were excluded. Discrepancies between reviewers were resolved through discussion and consensus.

Relevant data were extracted from each article according to the framework described in Wolfe et al., ${ }^{8}$ including author and publication details, WASH exposures, food exposures, study design features (e.g., matching of cases and controls), case and control definitions, number of cases and controls, geographic region, and demographic information. Quantitative data extracted included sample size, ORs, and 95\% Cls for each exposure. Typhoid case definitions for individual studies were examined to determine if they matched the WHO definition ("the isolation of $S$. Typhi from blood, bone marrow, or a specific anatomical lesion"). ${ }^{1}$ Water, sanitation, and hygieneand food-predicted protective and risk factors were defined, and individual exposures were categorized. Data were managed in Microsoft Excel 2010 (Redmond, WA).

Risk of bias appraisal. Two independent reviewers evaluated study quality using a risk of bias assessment tool adapted from the Quality Assessment Tool for Quantitative Studies by the Effective Public Health Practice Project ${ }^{17}$ by Wolfe et al. ${ }^{8,18,19}$ Criteria that monitored intervention implementation and long-term follow-up were removed, so the assessment can pertain specifically to case-control studies. Risk of bias was evaluated over five domains: 1) selection bias, 2) confounding, 3) incomplete data, 4) selective reporting, and 5) other bias (including misclassification bias, inappropriate use of statistical methods, and primarily selfreported data). We also evaluated whether information was sufficient to assess possible bias. Please note that analysis type was not included in bias assessment, and inappropriate use of bivariate or multivariable analysis would not have contributed to higher bias scores. Studies were scored as "low risk," "high risk," or "unclear" for each domain, and scores were added to generate a summary risk of bias. Studies scoring as "low risk" in 4-5 categories, 3 categories, and 1-2 categories were determined to have low, medium, and high summary bias, respectively.

Analysis. Data management and analysis were performed in Microsoft Excel (Redmond, WA) and Stata 15 (StataCorp LP, College Station, TX). Meta-analyses were performed using the ORs associated with exposures to determine a summary association for each of the predicted risk and protective factors identified during review using a Mantel-Haenszel random-effects analysis. Random-effects analysis was used because of the high heterogeneity between estimates from case-control studies. An $I^{2}$ test was used to formally quantify the amount of statistical heterogeneity observed, with significance determined using a Pearson chi-squared test. Funnel plots were generated to further illustrate systematic heterogeneity. Despite heterogeneity, meta-analysis was deemed appropriate because transmission pathways are similar across study contexts.
To assess the robustness of the overall associations, five sensitivity analyses were completed, by performing the same analysis including only exposures from the following: 1) studies assessed as low and medium summary risk of bias, 2) studies of any risk level that used the WHO case definition for confirmed typhoid cases, 3) low and medium risk studies that used the WHO case definition, 4) studies for which the recall period was $<2$ weeks, and 5) studies for which typical habits were used instead of a specific recall period.

\section{RESULTS}

Overall, 1,199 articles were identified in the initial search, 43 articles were included after reviewing title and abstract in the first screening, and 19 articles, including 22 individual casecontrol studies, were included after full-text review in the second screening (Supplemental Table 1, Figure 2). The articles represent studies from 12 countries: six from Indonesia, three each from India and Vietnam, two from Pakistan, and one each from Bangladesh, Myanmar, Nepal, Rwanda, Tajikistan, Uganda, Uzbekistan, and Zimbabwe. In the quality assessment, two studies ( $9 \%$ ) were categorized as having low risk of bias, one (5\%) as medium risk, and $19(86 \%)$ as high risk of bias. Of 22 studies, 11 (50\%) used the WHO definition of typhoid to identify cases (Supplemental Table 1). Among studies that did not use the WHO definition of typhoid, cases were defined by seropositive results, ${ }^{19-24}$ urine-positive results, ${ }^{24}$ clinical diagnosis, ${ }^{25-27}$ or clinical diagnosis in the setting of a negative malarial test. ${ }^{28}$

A total of 13 factors were identified under the five WASH groups defined as impacting typhoid transmission, including six predicted protective factors and seven predicted risk factors (Table 1). Unimproved water source, unsafe water management, and safe waste management were not included in meta-analysis because of lack of data ( $<5$ in each category). A total of nine factors were found to fall into the three food groups defined as impacting typhoid transmission, including two predicted protective factors and seven predicted risk factors (Table 2).

Water source. Water sources were categorized as improved (a predicted protective factor), unimproved, or surface water contact (predicted risk factors) based on the description of exposures in the text. Improved water source was defined according to JMP standards and includes sources that adequately protect water from outside contamination, such as piped water, tube wells, protected springs, and rainwater. ${ }^{9}$ The use of an improved water source is a predicted protective factor because protection from outside contamination is a barrier to fecal-oral contamination. ${ }^{18,29}$ Unimproved water source was defined based on JMP standards and was considered a predicted risk factor; however, only two identified exposures fell into this group, and meta-analysis was not performed. Contact with surface water was a predicted risk factor, as surface water is considered unimproved by the JMP. ${ }^{9}$

In meta-analysis, improved water source $(n=16)$ was associated with significantly lower odds of typhoid $(\mathrm{OR}=0.74,95 \%$ $\mathrm{Cl}=0.56-0.95)$ with a heterogeneity of $I^{2}=76 \%,{ }^{19,21,25,29-34}$ and surface water contact $(n=7)$ was significantly associated with higher odds of typhoid $(\mathrm{OR}=1.9,95 \% \mathrm{Cl}=1.4-2.5)$ with a heterogeneity of $I^{2}=32 \%$ (Table 3). ${ }^{21,23,33,35}$ In the sensitivity analysis of improved water source, direction and significance of 


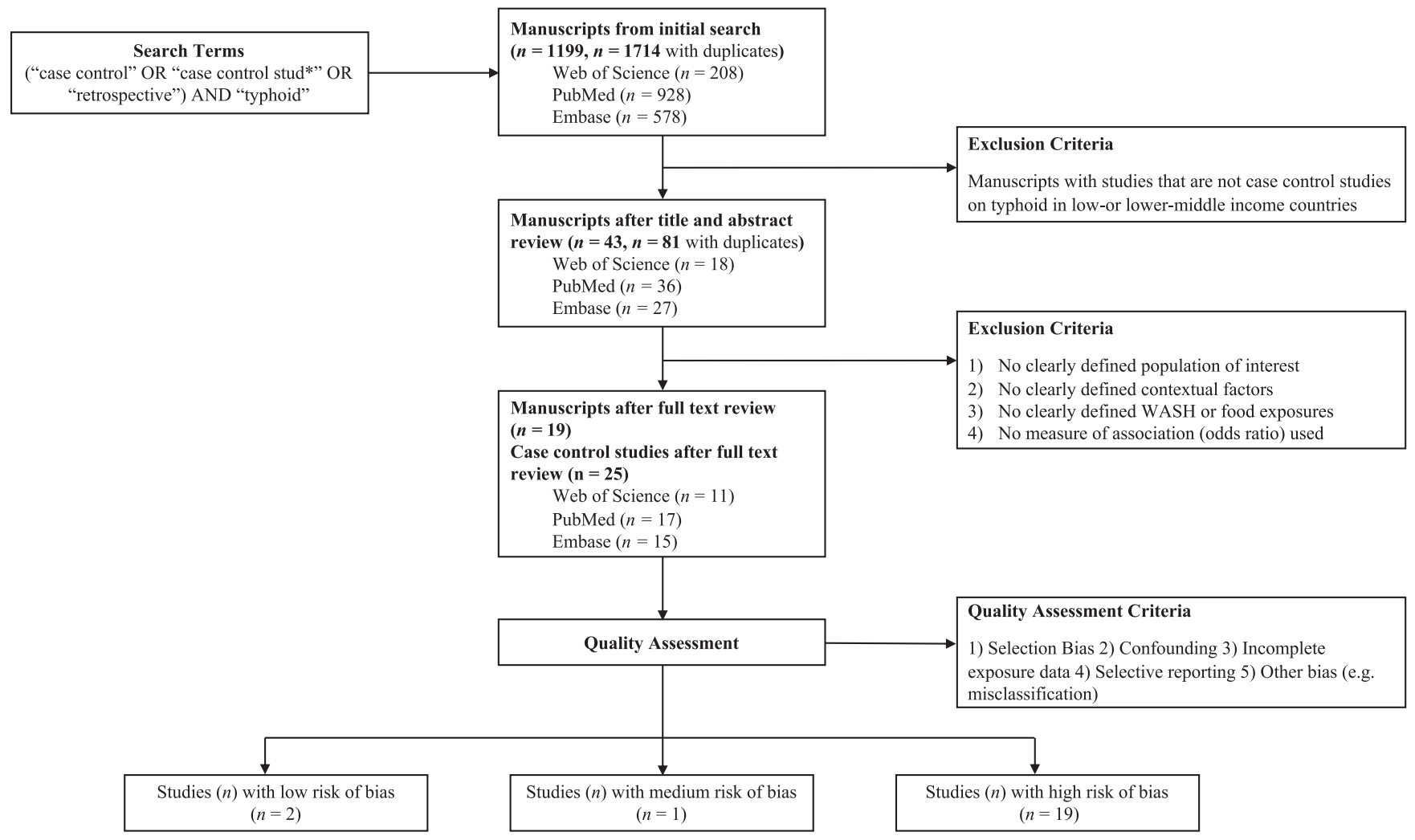

FIGURE 2. Study selection and quality assessment flowchart.

association were unchanged. Sensitivity analysis was not available for surface water contact (Supplemental Table 2).

Water treatment. Water treatment was defined as measures taken to make water safer to drink, including boiling, filtering, or treating with chlorine. Water treatment was a predicted protective factor, as these interventions are intended to remove or inactivate bacteria introduced through fecal-oral contamination. ${ }^{9}$ The lack of treatment was a predicted risk factor.

Treated water $(n=9)$ was significantly associated with lower odds of typhoid $(\mathrm{OR}=0.59,95 \% \mathrm{Cl}=0.45-0.75)$ with a heterogeneity of $I^{2}=39 \%,{ }^{19,21,24,27,31-33}$ whereas untreated water $(n=11)$ was significantly associated with higher odds of typhoid $(\mathrm{OR}=2.4,95 \% \mathrm{Cl}=2.0-2.9)$ and had a heterogeneity of $I^{2}=69 \%$ (Table 3, Figure 3). ${ }^{24,31-33,35,36}$ No changes in the direction of association or significance occurred in sensitivity analysis (Supplemental Table 2).

Water management. Water management includes practices related to water transport and storage. Safe water transport and storage prevent contamination of water through the use of protected containers, such as covered buckets and jerricans, that provide barriers to fecal-oral contamination. ${ }^{9}$ Safe practices were predicted protective factors. There were too few identified exposures $(n=4)$ to assess unsafe water management.

Safe water management $(n=9)$ was significantly associated with lower odds of typhoid $(\mathrm{OR}=0.67 ; 95 \% \mathrm{Cl}=0.51-0.86)$ with a heterogeneity of $P^{2}=84 \%$ (Table 3, Figure 4). ${ }^{19,21,26,27,30,33}$ No changes were observed in the direction or significance of safe water management in sensitivity analysis (Supplemental Table 2).

Sanitation. Sanitation practices were focused on household management of feces and classified into four categories: household latrine available/used, safe waste management, unsafe waste management, and open defecation. The five subcategories of the JMP sanitation ladder (open defecation, unimproved, limited, basic, and safely managed) were used to categorize exposures based on the descriptions presented in each study. Household latrine available/used was considered a predicted protective factor because it should provide a

TABLE 1

Predicted protective and risk factors, by WASH group

\begin{tabular}{lll}
\hline \multicolumn{1}{c}{ WASH group } & \multicolumn{1}{c}{ Predicted protective factors } & Predicted risk factors \\
\hline Water source & Improved water source & Unimproved water source \\
& & Surface water contact \\
Water treatment & Treated water & Untreated water \\
Water management & Safe water management & Unsafe water management \\
Sanitation & Household latrine available/used & Open defecation \\
Hygiene & Safe waste management & Unsafe waste management \\
\hline WASH = water, sanitation, and hygiene. & Good Hygiene & Lack of hygiene \\
\hline
\end{tabular}


TABLE 2

Predicted protective and risk factors, by food group

\begin{tabular}{lll}
\hline \multicolumn{1}{c}{ Food group } & Predicted protective factors & Predicted risk factors \\
\hline Food practices & Protective food practices & Risky food practices \\
Location of food and drink consumption & Food and drink at home & Food and drink outside home \\
Consumption of specific food & & Dairy \\
& & Ice cream \\
& & Fruit and juice \\
& & Shellfish and fish \\
\hline
\end{tabular}

barrier against fecal-oral transmission. Household latrine exposures lacked details regarding the type of latrine and its management, so although the category is considered protective, further categorization into the JMP subcategories of safely managed and basic latrine was not possible. Safe waste management was also considered a predicted protective factor because it separates feces from the environment and humans; however, no meta-analysis was performed because only one exposure fell into this category. Unsafe waste management included exposures, such as "burst sewer pipe at home," and unsanitary methods of emptying latrines (disposal into nearby stream). ${ }^{21,27}$ These exposures did not fit into the JMP ladder; however, descriptions within the studies indicated that the method of waste disposal failed to create a barrier between feces and humans; thus, this category was considered a predicted risk factor. ${ }^{10}$ Open defecation was considered a predicted risk factor because it does not create a barrier between feces and humans.

Household latrine available/used $(n=7)$ was not significantly associated with typhoid $(\mathrm{OR}=0.87,95 \% \mathrm{Cl}=0.68-1.1$, $I^{2}=79 \%$ ) (Table 3). ${ }^{21,24,30,32}$ Open defecation $(n=6)$ was not significantly associated with typhoid $(\mathrm{OR}=0.99,95 \% \mathrm{Cl}=$ $0.84-1.2, I^{2}=18 \%$ ) (Table 3). ${ }^{24,30,35}$ Unsafe waste management $(n=6)$ was significantly associated with increased odds of typhoid (OR $=1.6,95 \% \mathrm{Cl}=1.3-2.0, l^{2}=55 \%$ ) (Table 3, Figure 5). ${ }^{21,24,27,36}$ In sensitivity analysis, the association between household latrine available/used became significant when studies with a recall period of $<2$ weeks $(n=6)$ were included $(\mathrm{OR}=0.50,95 \% \mathrm{Cl}=0.34-0.75)$. Sensitivity analysis was not available for open defecation or unsafe waste management (Supplemental Table 2).

Hygiene. Hygiene includes behaviors that promote cleanliness, such as handwashing with soap and water. ${ }^{22,23}$ Hygiene is defined by the JMP as "conditions and practices that help maintain health and prevent spread of disease including handwashing,... and food hygiene."10 Signs of good hygiene (e.g., handwashing and use of soap and water) were predicted protective factors because hygiene creates a barrier between fecal material and the new host. A lack of hygiene facilitates the spread of disease and is a predicted risk factor. ${ }^{10,24}$ We considered food exposures separately in this review.

Lack of hygiene $(n=15)$ was significantly associated with higher odds of typhoid $(\mathrm{OR}=2.2,95 \% \mathrm{Cl}=1.9-2.6)$ with a heterogeneity of $I^{2}=36 \%$ (Table 3, Figure 6). ${ }^{20,22,24-26,34,37}$ Good hygiene $\left(n=8, \mathrm{OR}=0.52,95 \% \mathrm{Cl}=0.40-0.67, I^{2}=82 \%\right)$ was significantly associated with lower odds of typhoid (Table 3, Figure 6). ${ }^{19,23,24,32}$ There was no change in the direction of association or significance in the sensitivity analysis of lack of hygiene. Sensitivity analysis was unavailable for good hygiene (Supplemental Table 2).

Food practices. Safe food practices are behaviors that reduce the likelihood of disease being passed on through ingestion of food. Exposures were categorized as protective food practices or risky food practices based on the WHO Safer Food guidelines. ${ }^{12}$

The protective food practices category $(n=8)$ was significantly associated with lower odds of typhoid (OR $=0.74,95 \%$ $\mathrm{Cl}=0.55-1.0)$ with a heterogeneity of $I^{2}=80 \%,{ }^{19,24,25,33}$ whereas the risky food practices category $(n=15)$ was significantly associated with higher odds of typhoid (OR $=1.7$, $95 \% \mathrm{Cl}=1.5-2.0, I^{2}=63 \%$ ) (Table 4, Figure 7$) .^{20,22,24,34}$ Neither the direction nor the significance of the association of protective or risky food practices changed in sensitivity analysis (Supplemental Table 3).

Location of food and drink consumption. The location of food and drink consumption was detailed in many of the articles, with in-home consumption and consumption outside the home emerging as two overarching categories. Food and drink consumed at home had too few exposures $(n=4)$ to be included in meta-analysis. Food and drink consumed outside home was considered a predicted risk factor for acquiring typhoid as consumers have less control over food practices and street-vended food, and food available outside home has been shown to confer risk of acquiring other foodborne diseases. ${ }^{38-40}$ Furthermore, each study included this exposure as a means of determining the source of outbreak or as a point of transmission in endemics. Several factors in this

TABLE 3

Odds ratios and $95 \%$ Cls from predicted protective and risk factors, by WASH group

\begin{tabular}{|c|c|c|c|c|c|c|c|}
\hline Predicted protective factors & $N$ (exposures) & OR $(95 \% \mathrm{Cl})$ & $l^{2}$ & Predicted risk factors & $N$ (exposures) & OR $(95 \% \mathrm{Cl})$ & $l^{2}$ \\
\hline Improved water source & 16 & $0.73(0.56,0.95)$ & $76 \% *$ & Surface water contact & 7 & $1.85(1.37,2.49)$ & $32 \%$ \\
\hline Treated water & 9 & $0.59(0.45,0.75)$ & $39 \%$ & Untreated water & 12 & $2.39(1.95,2.93)$ & $69 \%$ * \\
\hline Safe water management & 9 & $0.67(0.51,0.86)$ & $84 \% *$ & & & & \\
\hline \multirow[t]{2}{*}{ Household latrine available/used } & 7 & $0.87(0.68,1.11)$ & $79 \% *$ & Open defecation & 6 & $0.99(0.84,1.18)$ & $18 \%$ \\
\hline & & & & Unsafe waste management & 6 & $1.56(1.25,1.95)$ & $55 \%$ * \\
\hline Good hygiene & 8 & $0.52(0.40,0.67)$ & $82 \%$ * & Lack of hygiene & 15 & $2.20(1.86,2.60)$ & $36 \%$ \\
\hline
\end{tabular}

WASH = water, sanitation, and hygiene; $\mathrm{OR}=$ odds ratio.

* Heterogeneity significant at the $P<0.05$ level ( $l^{2}$ test of heterogeneity with significance determined with Pearson's chi-squared test). 


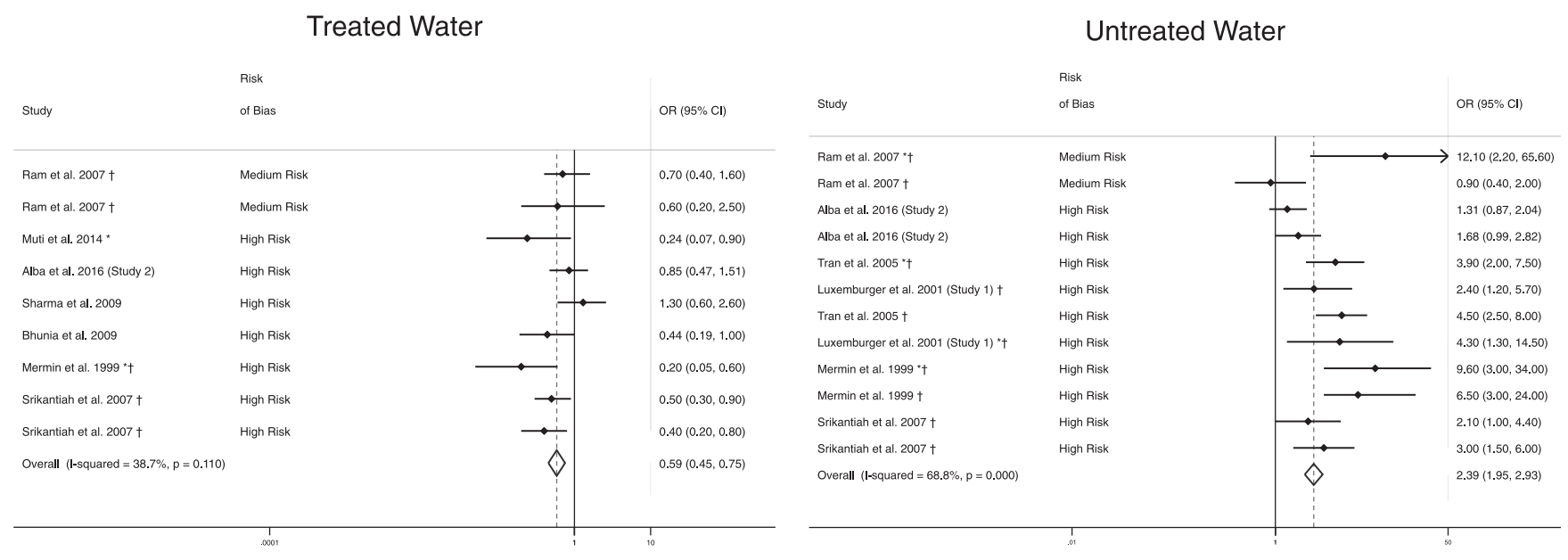

FIGURE 3. Meta-analysis of the association between water treatment and typhoid, including water treatment and no water treatment. †Study used the WHO case definition for typhoid. *Odds ratio reported from multivariate analysis.

category included food and drink from street vendors, restaurants, work, mosques, and school.

Food and drink consumed outside home $(n=39)$ was significantly associated with higher odds of typhoid (OR $=1.6$, $95 \% \mathrm{Cl}=1.4-1.8$ ) with a heterogeneity of $I^{2}=72 \%$ (Table 4 , Figure 8). ${ }^{19,20,24-26,28-37}$ Neither the direction nor the significance of association of food and drink consumed outside the home changed in sensitivity analysis (Supplemental Table 3).

Consumption of specific foods. Case-control studies often investigate specific foods suspected to be the source or vehicle of transmission of an outbreak. Dairy, ice cream, fruit

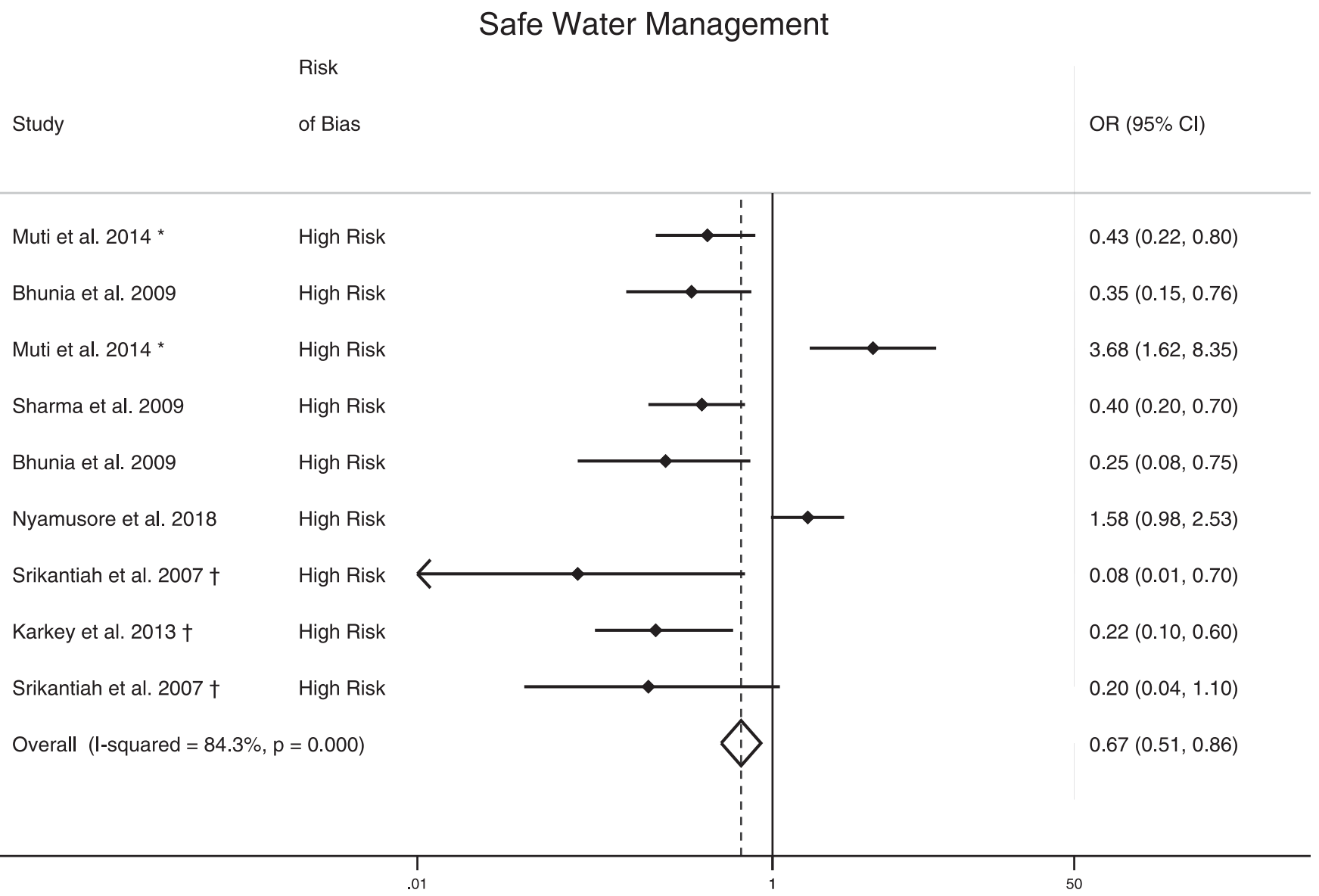

FIGURE 4. Meta-analysis of the association between safe water management and typhoid. †Study used the WHO case definition for typhoid. *Odds ratio reported from multivariate analysis. 


\section{Unsafe Waste Management}

Risk

Study

of Bias

OR $(95 \% \mathrm{Cl})$

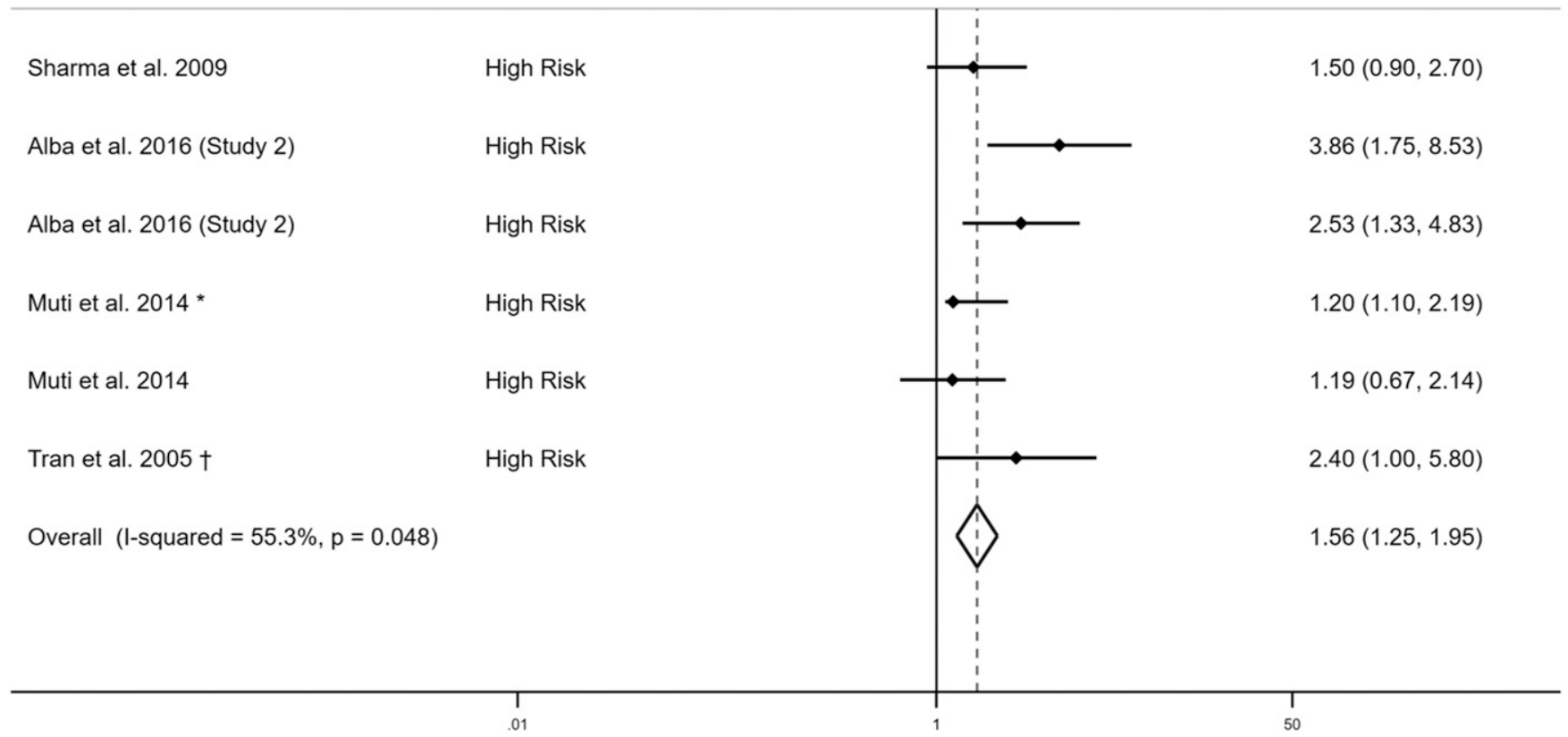

FIGURE 5. Meta-analysis of the association between unsafe waste management and typhoid. †Study used the WHO case definition for typhoid. *Odds ratio reported from multivariate analysis.

and juice, shellfish and fish, and vegetables were specific food categories that included enough exposures to undergo metaanalysis. Ice cream was assessed independently from dairy because of previous evidence linking ice cream to typhoid outbreaks. ${ }^{41,42}$

Dairy consumption ( $n=12)$ was significantly associated with higher odds of typhoid $(\mathrm{OR}=1.4,95 \% \mathrm{Cl}=1.1-1.7$, $\left.l^{2}=75 \%\right),{ }^{19,21,29-33}$ as was ice cream consumption $(n=10$, $\left.\mathrm{OR}=1.5,95 \% \mathrm{Cl}=1.2-1.9, I^{2}=31 \%\right)^{19,21,29,32,33,35,37}$ and fruit and juice consumptions $(n=21, \mathrm{OR}=1.5,95 \% \mathrm{Cl}=1.2-1.8$, $I^{2}=52 \%$ ) (Table 4). ${ }^{21,28,29,31-33}$ Shellfish and fish consumption $(n=9)$ was not significantly associated with higher or lower odds of typhoid (OR $=0.98,95 \% \mathrm{Cl}=0.79-1.2$, $\left.I^{2}=75 \%\right) .^{29,35,36}$ Vegetable consumption $(n=14)$ was

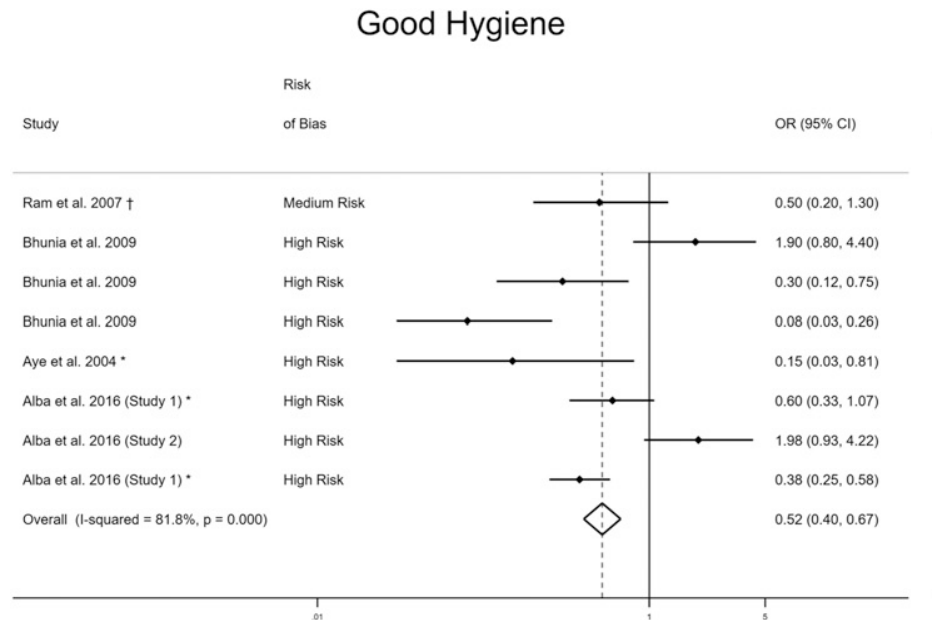

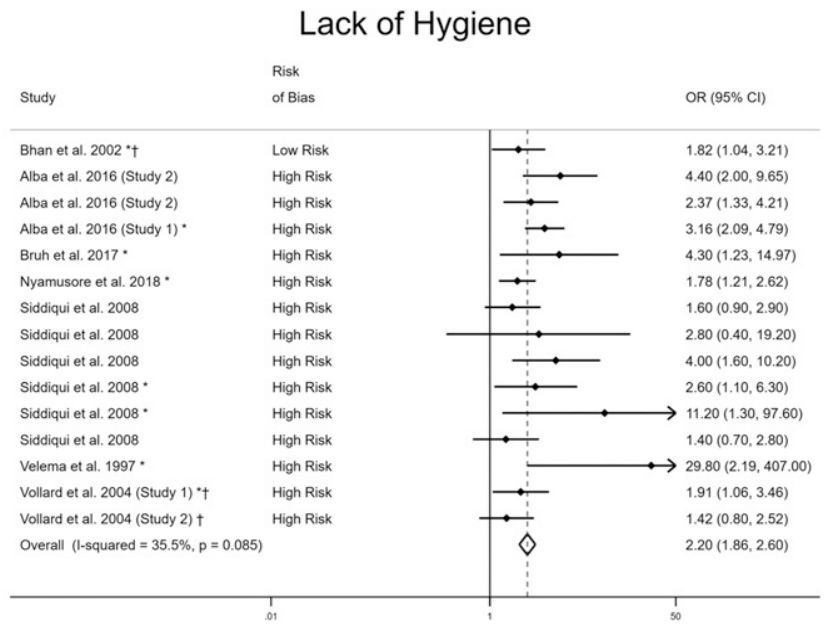

FIGURE 6. Meta-analysis of the association between hygiene and typhoid, including good hygiene and lack of hygiene. †Study used the WHO case definition for typhoid. *Odds ratio reported from multivariate analysis. 
TABLE 4

Odds ratios and $95 \%$ Cls from predicted protective and risk factors, by food group

\begin{tabular}{|c|c|c|c|}
\hline & $N$ (exposures) & OR $(95 \% \mathrm{Cl})$ & $1^{2}$ \\
\hline \multicolumn{4}{|l|}{ Predicted protective factors } \\
\hline Protective food practices & 8 & $0.74(0.55,1.00)$ & $80 \% *$ \\
\hline \multicolumn{4}{|l|}{ Predicted risk factors } \\
\hline Risky food practices & 15 & $1.71(1.45,2.03)$ & $63 \%{ }^{*}$ \\
\hline Food and drink outside home & 39 & $1.62(1.44,1.83)$ & $72 \%$ * \\
\hline \multicolumn{4}{|c|}{ Predicted risk factors (specific foods) } \\
\hline Dairy & 12 & $1.38(1.12,1.71)$ & $75 \% *$ \\
\hline Ice cream & 10 & $1.49(1.19,1.86)$ & $31 \%$ \\
\hline Fruit and juice & 21 & $1.46(1.22,1.75)$ & $52 \%{ }^{*}$ \\
\hline Shellfish and fish & 9 & $0.98(0.79,1.22)$ & $75 \%$ * \\
\hline Vegetables & 14 & $1.13(0.97,1.31)$ & $63 \%{ }^{*}$ \\
\hline
\end{tabular}

not significantly associated with higher or lower odds of typhoid $\left(\mathrm{OR}=1.1,95 \% \mathrm{Cl}=0.97-1.3, \mathrm{I}^{2}=63 \%\right)$ (Table 4). ${ }^{19,21,24,29,31-33}$

In sensitivity analysis, no association with typhoid was observed for dairy consumption, including only studies that use the $\mathrm{WHO}$ definition of typhoid $(n=8, \mathrm{OR}=0.88,95 \% \mathrm{Cl}=$ 0.67-1.2) (Supplemental Table 3). No difference in association or significance was observed in sensitivity analyses of ice cream consumption, fruit and juice consumption, and shellfish and fish consumption. Vegetable consumption became significantly associated with the higher odds of typhoid among studies using a recall period of $<2$ weeks $(n=11$, OR $=1.4$, $95 \% \mathrm{Cl}=1.1-1.7$ ) (Supplemental Table 3).

\section{DISCUSSION}

We conducted a systematic review and meta-analysis of case-control studies to investigate the relationship among WASH and food exposures and typhoid. In total, 19 articles describing 22 case-control studies were included in the review. Overall, WASH exposures were grouped into five predicted risk factors and five predicted protective factors (Table 1). Four (80\%) of five predicted risk factors were associated with the increased odds of typhoid $\left(\mathrm{OR}=1.6-2.4, I^{2}=\right.$ $32-69 \%)$, and $4(80 \%)$ of five predicted protective factors were associated with the lower odds of typhoid transmission
$\left(\mathrm{OR}=0.52-0.73, l^{2}=39-84 \%\right)$ (Table 3). Good hygiene and water treatment were most strongly associated with protection from typhoid ( $\mathrm{OR}=0.52$ and 0.59 , respectively), whereas poor hygiene and untreated water were most strongly associated with the risk of typhoid $(O R=2.2$ and 2.4, respectively). Of the three sanitation factors, only unsafe waste management was significantly associated with typhoid $(\mathrm{OR}=1.6,95 \% \mathrm{Cl}=1.3-2.0)$.

The single predicted protective food factor (protective food practices) was significantly associated with lower odds of typhoid $\left(\mathrm{OR}=0.74, I^{2}=80 \%\right)$, and both predicted food management risk factors (risky food practices and consuming food or drink outside the home) were associated with a significantly higher odds of typhoid (OR = 1.6-1.7, $I^{2}=63-72 \%$ ) (Table 4). Three specific foods (dairy, ice cream, and fruits and juices) were significantly associated with typhoid $\left(\mathrm{OR}=1.4-1.5, I^{2}=\right.$ $31-75 \%)$. In sensitivity analyses, only $3(8 \%)$ of 26 summary associations changed significance from the original analysis, indicating robustness of results; however, only one or fewer sensitivity analyses could be performed in 11 (61\%) of 18 exposures groups because of limited study numbers in the exposure categories.

Our results suggest that behaviors focused within the household, including hygiene, food safety, and water treatment, are interventions that may reduce typhoid transmission by reducing human contact with fecally contaminated food

\section{Protective Food Practices}

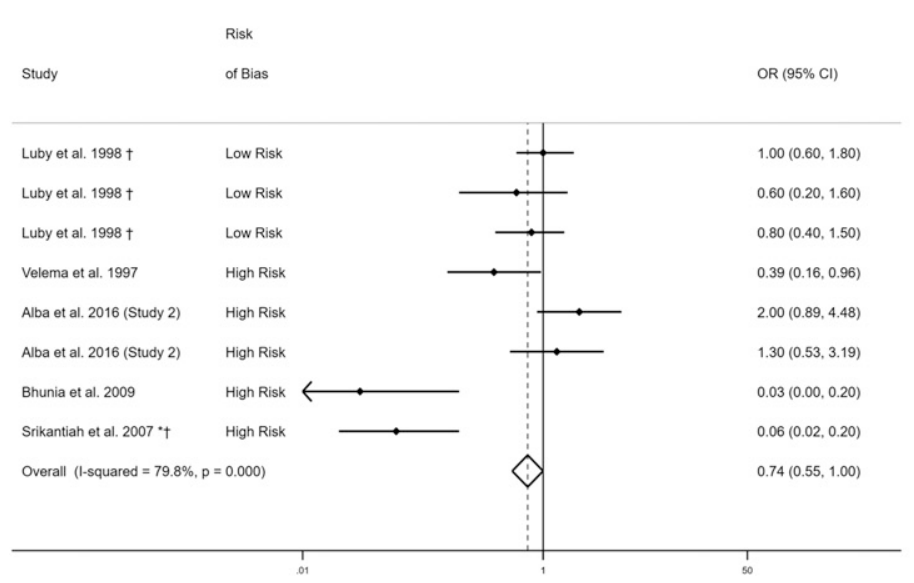

\section{Risky Food Practices}

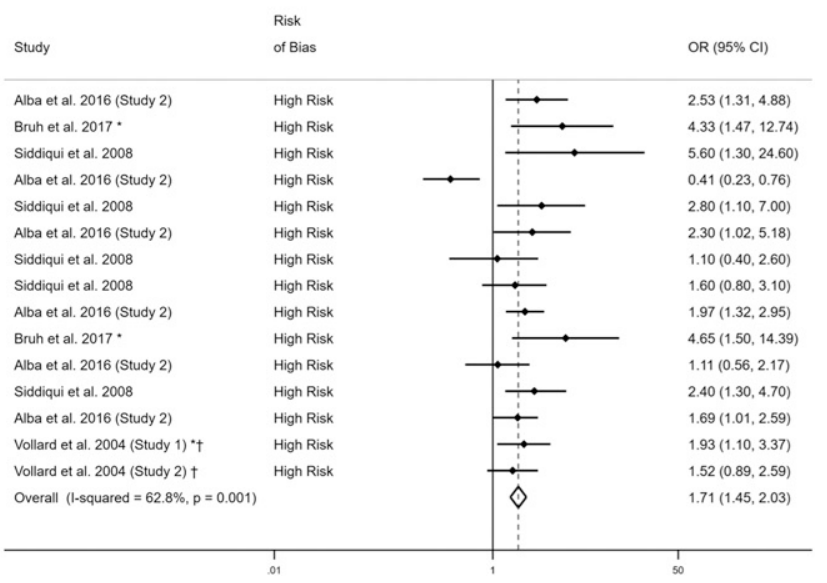

FIGURE 7. Meta-analysis of the association between food practices and typhoid, including protective food practices and risky food practices. †Study used the WHO case definition for typhoid. ${ }^{*}$ Odds ratio reported from multivariate analysis. 


\section{Food and Drink Outside of Home}

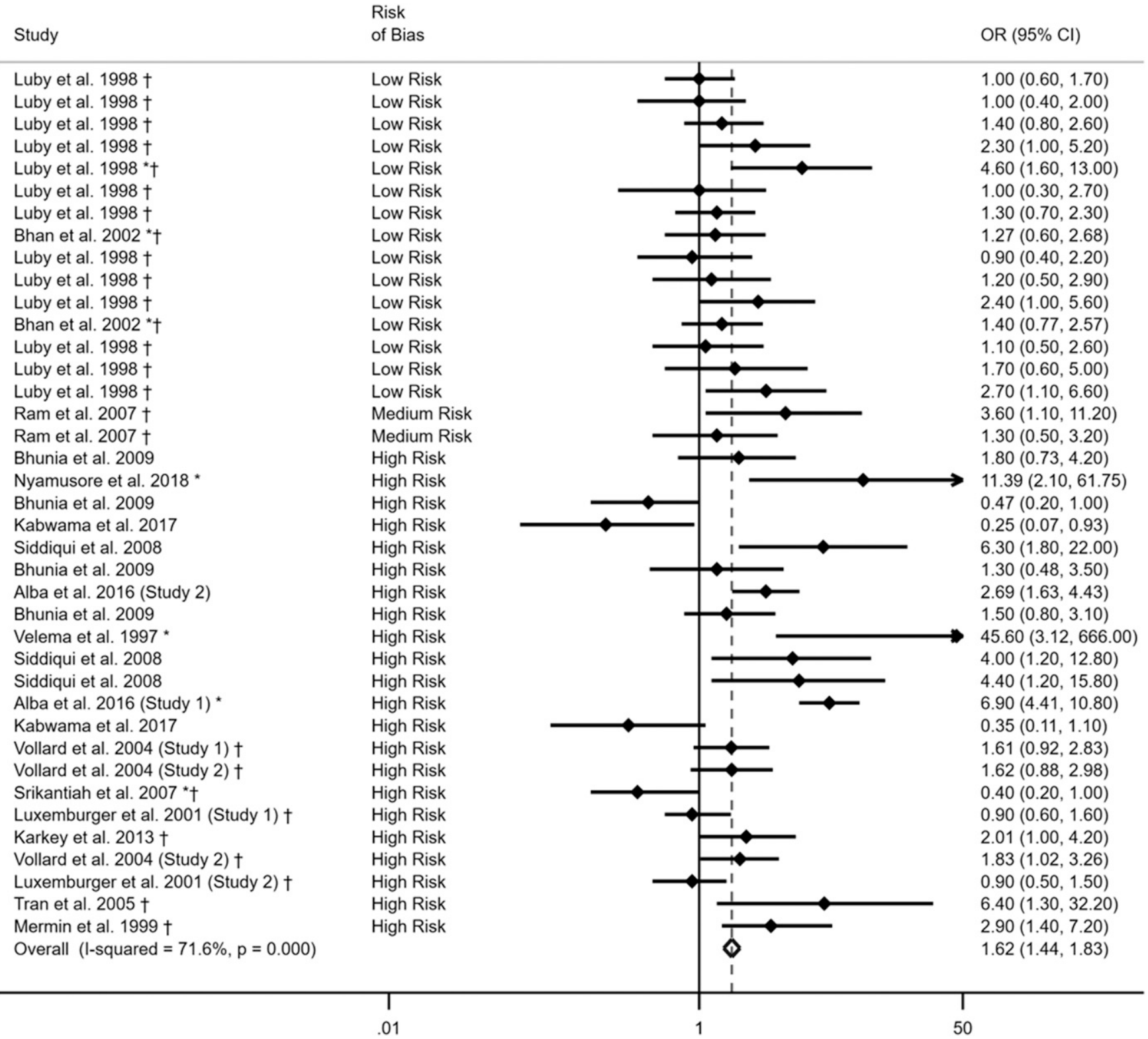

FIGURE 8. Meta-analysis of the association between food and drink outside home and typhoid. $\uparrow$ Study used the WHO case definition for typhoid. ${ }^{*}$ Odds ratio reported from multivariate analysis.

and water in homes. These were practiced much less frequently in case households than in households of controls. Because persons with typhoid fever may shed bacteria before the onset of symptoms and after their resolution, these interventions should be practiced by all individuals in a household; however, their adoption and maintenance may require behavior change, which may make them challenging to implement and sustain. In addition, we do not intend in any way to "blame" households or individuals for short-cycle typhoid transmission. We are summarizing available evidence from case-control studies available, to inform recommendations for protective practices by households and individuals that could contribute to the containment of typhoid outbreaks once they occur.
Foodborne transmission of typhoid has been welldocumented, and our results highlight the need to study food practices and specific foods as contributing to typhoid transmission. ${ }^{43}$ Our study included a group of food practices that were collectively predicted to be protective, including reheating cooked food before serving, cleaning the kitchen regularly (practices that would kill or reduce the load of Typhi bacteria), using a spoon to serve food (creating a barrier between a carrier and healthy consumers), routinely washing raw food before eating (reducing the risk of exposure from "upstream" contamination), and using a refrigerator (which may reduce the risk of Typhi multiplication). ${ }^{44}$ However, a limitation is that we were unable to identify in this meta-analysisspecific practices that were most likely to be protective. For 
example, previous data suggest that Typhi can persist even at low temperatures in certain foods (e.g., ice cream), ${ }^{45}$ casting in doubt the use of a refrigerator itself as a fully protective factor. These results highlight the need for more data on typhoid protection and risk associated with specific food practices in outbreak and endemic settings.

Previous studies have suggested that street-vended food and food available outside the home is a risk factor for acquiring foodborne diseases, and our study results support this finding for typhoid. ${ }^{38-40}$ In fact, because of the potential for lifelong asymptomatic carriage and shedding of Typhi, food handlers may transmit disease to a wide customer base over a prolonged period. ${ }^{46-49}$ Although specific foods, including dairy, ice cream, and fruit and juice, were significantly associated with increased risk of typhoid transmission, we were unable to take into account how these foods were prepared or where they were consumed. We suggest that future casecontrol studies expand on these results by comparing the risks associated with consuming specific foods outside the home versus the risks of consuming those same foods in the home.

Last, factors commonly associated with other faeco-orally transmitted diseases with outbreak potential, such as cholera, were not associated with typhoid fever transmission, including consumption of fish and use of a latrine versus open defecation. ${ }^{8,50,51}$ The differences in the microbiologic and environmental characteristics of Typhi and toxigenic Vibrio cholerae serogroups $\mathrm{O} 1$ and $\mathrm{O} 139$ are likely responsible in large part for these differences (e.g., cholera has an environmental reservoir, whereas typhoid likely only has a human reservoir) and highlights the need to consider each disease separately and prioritize specific intervention efforts accordingly. In the case of typhoid, guidelines regarding household hygiene practices, water treatment, and caution when consuming food from outside the home should be emphasized.

One challenge in completing this review was that WASH interventions were often incompletely described, making them difficult to assess and categorize, as was the case for sanitation. Undescribed differences between the included studies likely contributed to the high degree of heterogeneity we observed in our results. A previous study summarizing WASH factors in cholera outbreaks also reported this challenge. $^{8}$ More detailed information on study design and implementation of interventions in case-control studies will make it easier for future meta-analyses to identify specific risk and protective factors more precisely.

This study had several limitations. Case-control studies are subject to a risk of bias in the selection of cases and controls, to courtesy and recall bias, and to bias in the creation and implementation of the exposure questionnaires. Robust and standardized questionnaires will capture the full range of potential exposures included in this review; however, some case-control studies focus on a subset of these exposure pathways. This could lead to over- or underreporting bias in results, masking important exposure pathways. Only half of the studies (11 of 22) defined typhoid cases by isolation and identification of Typhi cultured from blood, stool, urine, or marrow, whereas the remainder defined them by clinical symptoms, serological testing, or both, which could lead to the dilution of estimated effects through misclassification. Some exposures were reported few times, meaning some practices could not be evaluated and necessitating the creation of broad categories to estimate the effect of risky and predictive food practices. It is hoped that more consistent investigation and reporting of protective and risky WASH factors, as recommended earlier, will reduce these biases in the future. In addition, variations in population immunity, particularly in endemic settings, may have contributed to variability observed in interventions. ${ }^{52,53}$

In addition, 19 (86\%) of the 22 case-control studies were classified as "high risk of bias," and when these were removed during sensitivity analysis, only two (11\%) of 18 exposures could be examined. This suggests that although in general the results are relatively robust (i.e., they change little when subjected to various sensitivity analyses), caution should be used in their interpretation, as there are more potential sources of bias than we were unable to control for statistically. Although every effort was made to place factors in the correct category, lack of intervention details may have led to misclassification. We are able to describe factors reported but are not able to compare this with factors measured-only two (9\%) studies provided detail on questionnaires, whereas 12 (55\%) studies failed to report all investigated risk factors, making it difficult to assess reporting bias. Our analysis was limited to peerreviewed articles written in English, and publication bias may have led to underreporting of null findings, which could have affected results by making summary ORs more extreme. An additional limitation of this study is that there were insufficient data and inconsistent approaches for conducting multivariate analysis to conduct sensitivity analysis in which bivariate results are removed. Again, it is hoped that more consistent reporting of protective and risky WASH factors, as recommended in the article, will allow this type of analysis in the future.

Although we believe that a meta-analysis to summarize these data is useful to highlight some of the dominant Typhi transmission pathways and identify underemphasized areas for research and intervention, we acknowledge that the included studies are heterogenous, and dynamics influencing transmission can vary substantially from one outbreak to another. We note not only the important patterns in these results but also the diversity WASH and food risk and protective factors that are associated with typhoid in different contexts. Thus, we are also cautious about overinterpretation of these data. Ideally, we would have performed a multivariate metaanalysis that would account for effect modification; however, low study quality overall, with insufficient data on exposures, interventions, and inter-study correlations precluded that possibility. Similarly, because of the quality and heterogeneity of the data, we are not comfortable calculating a population attributable fraction of typhoid associated with each of these factors.

Our review highlights the potential for household hygiene interventions and water treatment to reduce disease in LMIC with endemic and outbreak typhoid. Results also suggest that further investigation is needed into the association among typhoid and protective food practices and the consumption of specific foods outside and within the home. The differences in the pattern of association among WASH and food factors and disease between typhoid and other diseases, such as cholera, emphasize the need to tailor interventions specific to typhoid rather than simply implementing factors that have been successful in other waterborne and foodborne diseases. We also note that although this review addresses factors influencing 
transmission in a contaminated environment, further work is necessary to address behaviors and infrastructure that result in contaminated water and food supplies at a macro-system level beyond the household. Finally, the future inclusion of detailed WASH and food exposures in case-control studies will help address the limitations described in our review and inform targeted interventions.

Received June 26, 2019. Accepted for publication February 4, 2020.

Published online July 20, 2020.

Note: Supplemental tables appear at www.ajtmh.org.

Acknowledgments: We would like to thank Amy Lapidow and Elizabeth Settoducato, Tufts librarians, for their assistance in developing the search strategy.

Financial support: S. B. was supported by the William Harold, MD, Medical Student Research Fellowship through Tufts University School of Medicine.

Disclaimer: The findings and conclusions in this report are those of the authors and do not necessarily represent the official position of the Centers for Disease Control and Prevention.

Authors' addresses: Sarah Brockett, Tufts University School of Medicine, Boston, MA, E-mail: sarah.brockett@tufts.edu. Marlene K. Wolfe, Department of Civil and Environmental Engineering, Stanford University, E-mail: marlene.wolfe@stanford.edu. Asa Hamot, Concord Academy, Concord, MA, E-mail: asa.hamot@concordacademy.org. Grace D. Appiah and Eric D. Mintz, Centers for Disease Control and Prevention, Atlanta, GA, E-mails:ydg3@cdc.gov and edm1@cdc.gov.

\section{REFERENCES}

1. Acosta $C$ et al., 2003. Background document: The diagnosis, Treatment and Prevention of Typhoid fever. Available at: http:// www.who.int/rpc/TFGuideWHO.pdf. Accessed June 1, 2018.

2. Spano S, 2016. Mechanisms of Salmonella Typhi host restriction. Adv Exp Med Biol 915: 283-294.

3. Mogasale V, Maskery B, Ochiai RL, Lee JS, Mogasale VV, Ramani E, Kim YE, Park JK, Wierzba TF, 2014. Burden of typhoid fever in low-income and middle-income countries: a systematic, literature-based update with risk-factor adjustment. Lancet Glob Health 2: e570-e580.

4. Antillon M, Warren JL, Crawford FW, Weinberger DM, Kurum E, Pak GD, Marks F, Pitzer VE, 2017. The burden of typhoid fever in low- and middle-income countries: a meta-regression approach. PLoS Negl Trop Dis 11: e0005376.

5. Stanway $\mathrm{J}$ et al., 2019. The global burden of typhoid and paratyphoid fevers: a systematic analysis for the Global Burden of Disease Study 2017. Lancet Infect Dis 19: 369-381.

6. Crump JA, Sjolund-Karlsson M, Gordon MA, Parry CM, 2015. Epidemiology, clinical presentation, laboratory diagnosis, antimicrobial resistance, and antimicrobial management of invasive Salmonella infections. Clin Microbiol Rev 28: 901-937.

7. Dougan G, Baker S, 2014. Salmonella enterica serovar Typhi and the pathogenesis of typhoid fever. Annu Rev Microbiol 68: 317-336.

8. Wolfe M, Kaur M, Yates T, Woodin M, Lantagne D, 2018. A systematic review and meta-analysis of the association between water, sanitation, and hygiene exposures and cholera in casecontrol studies. Am J Trop Med Hyg 99: 534-545.

9. WHO/UNICEF Joint Monitoring Programme: Sanitation, 2018. Sanitation. Available at: https://washdata.org/monitoring/ sanitation. Accessed June 1, 2018.

10. WHO/UNICEF Joint Monitoring Programme: Hygiene, 2018. Hygiene. Available at: https://washdata.org/monitoring/hygiene. Accessed June 1, 2018.

11. The Himmelfarb Health Sciences Library, 2011. Study Design 101 - Case Control Study. Available at: https://himmelfarb. gwu.edu/tutorials/studydesign101/casecontrols.html. Accessed June 10, 2018.
12. Yates T, Vujcic JA, Joseph ML, Galandat K, Lantagne D, 2018. Efficacy and effectiveness of water, sanitation, and hygiene interventions in emergencies in low- and middle-income countries: a systematic review. Waterlines 38, 31-65.

13. PRISMA, 2015. PRISMA: Transparent Reporting of Systematic Reviews and Meta-Analyses. Available at: http://www.prsmastatement.org/. Accessed June 1, 2018.

14. PROSPERO, 2018. PROSPERO: International Prospective Register of Systematic Reviews. Available at: https://www.crd.york.ac.uk/ prospero/. Accessed June 18, 2018.

15. O'Connor DA, Green S, Higgins JP eds., 2011. Cochrane Handbook for Systematic Reviews of Interventions. Available at: https://handbook-5-1.cochrane.org/chapter_5/5_defining_ the_review_question_and_developing_criteria for.htm.

16. Bakach I, Just MR, Gambhir M, Fung IC-H, 2015. Typhoid transmission: a historical perspective on mathematical model development. Trans R Soc Trop Med Hyg 109: 679-689.

17. Effective Public Health Practice Project, 2017. EPHPP Quality Assessment Tool for Quantitative Studies. Available at: http:// www.ephpp.ca/tools.html. Accessed May 3, 2017.

18. Fewtrell L, Kaufmann RB, Kay D, Enanoria W, Haller L, Colford JM Jr., 2005. Water, sanitation, and hygiene interventions to reduce diarrhoea in less developed countries: a systematic review and meta-analysis. Lancet Infect Dis 5: 42-52.

19. Bhunia R, Hutin Y, Ramakrishnan R, Pal N, Sen T, Murhekar M, 2009. A typhoid fever outbreak in a slum of South Dumdum municipality, West Bengal, India, 2007: evidence for foodborne and waterborne transmission. BMC Public Health 9: 115.

20. Siddiqui FJ, Haider SR, Bhutta ZA, 2008. Risk factors for typhoid fever in children in squatter settlements of Karachi: a nested case-control study. J Infect Public Heal 1: 113-120.

21. Sharma PK, Ramakrishnan R, Hutin Y, Manickam P, Gupte MD, 2009. Risk factors for typhoid in Darjeeling, West Bengal, India: evidence for practical action. Trop Med Int Health 14: 696-702.

22. Bruh RH, Hadisaputro S, Budhi K, Sakundarno M, Widjanarko B, 2017. Host risk factors for typhoid fever in children (A case study of north sumatera province). Adv Sci Lett 23: 3541-3543.

23. Aye TT, Siriarayapon $P, 2004$. Typhoid fever outbreak in madaya township, mandalay division, Myanmar, september 2000. J Med Assoc Thai 87: 395-399.

24. Alba $S$ et al., 2016. Risk factors of typhoid infection in the Indonesian archipelago. Plos One 11: e0155286.

25. Velema JP, vanWijnen G, Bult $P$, vanNaerssen T, Jota S, 1997. Typhoid fever in Ujung Pandang, Indonesia-high-risk groups and high-risk behaviours. Trop Med Int Health 2: 1088-1094.

26. Nyamusore J, Nahimana MR, Ngoc CT, Olu O, Isiaka A, Ndahindwa V, Dassanayake L, Rusanganwa A, 2018. Risk factors for transmission of Salmonella Typhi in Mahama refugee camp, Rwanda: a matched case-control study. Pan Afr Med J 29: 148.

27. Muti M, Gombe N, Tshimanga M, Takundwa L, Bangure D, Mungofa S, Chonzi P, 2014. Typhoid outbreak investigation in Dzivaresekwa, suburb of Harare city, Zimbabwe, 2011. Pan Afr Med J 18: 309.

28. Kabwama SN et al., 2017. A large and persistent outbreak of typhoid fever caused by consuming contaminated water and street-vended beverages: Kampala, Uganda, January-June 2015. BMC Public Health 17: 23.

29. Luby SP, Faizan MK, Fisher-Hoch SP, Syed A, Mintz ED, Bhutta ZA, McCormick JB, 1998. Risk factors for typhoid fever in an endemic setting, Karachi, Pakistan. Epidemiol Infect 120: 129-138.

30. Karkey A et al., 2013. Differential epidemiology of Salmonella Typhi and Paratyphi A in Kathmandu, Nepal: a matched case control investigation in a highly endemic enteric fever setting. PLoS Negl Trop Dis 7: e2391.

31. Mermin JH et al., 1999. A massive epidemic of multidrug-resistant typhoid fever in Tajikistan associated with consumption of municipal water. J Infect Dis 179: 1416-1422.

32. Ram PK, Naheed A, Brooks WA, Hossain MA, Mintz ED, Breiman RF, Luby SP, 2007. Risk factors for typhoid fever in a slum in Dhaka, Bangladesh. Epidemiol Infect 135: 458-465.

33. Srikantiah P, Vafokulov S, Luby SP, Ishmail T, Earhart K, Khodjaev N, Jennings G, Crump JA, Mahoney FJ, 2007. Epidemiology 
and risk factors for endemic typhoid fever in Uzbekistan. Trop Med Int Health 12: 838-847.

34. Vollaard AM, Ali S, van Asten H, Widjaja S, Visser LG, Surjadi C, van Dissel JT, 2004. Risk factors for typhoid and paratyphoid fever in Jakarta, Indonesia. JAMA 291: 2607-2615.

35. Luxemburger C, Chau MC, Mai NL, Wain J, Tran TH, Simpson JA, Le HK, Nguyen TT, White NJ, Farrar JJ, 2001. Risk factors for typhoid fever in the Mekong delta, southern Viet Nam: a casecontrol study. Trans $R$ Soc Trop Med Hyg 95: 19-23.

36. Tran HH, Bjune G, Nguyen BM, Rottingen JA, Grais RF, Guerin PJ, 2005. Risk factors associated with typhoid fever in Son La province, northern Vietnam. Trans $R$ Soc Trop Med Hyg 99: 819-826.

37. Bhan MK, Bahl R, Sazawal S, Sinha A, Kumar R, Mahalanabis D, Clemens JD, 2002. Association between Helicobacter pylori infection and increased risk of typhoid fever. J Infect Dis 186: 1857-1860.

38. Al Mamun M, Rahman SM, Turin TC, 2013. Microbiological quality of selected street food items vended by school-based street food vendors in Dhaka, Bangladesh. Int J Food Microbiol 166: 413-418.

39. Estrada-Garcia T, Lopez-Saucedo C, Zamarripa-Ayala B, Thompson MR, Gutierrez-Cogco L, Mancera-Martinez A, Escobar-Gutierrez A, 2004. Prevalence of Escherichia coli and Salmonella spp. in street-vended food of open markets (tianguis) and general hygienic and trading practices in Mexico City. Epidemiol Infect 132: 1181-1184.

40. Sharma I, Mazumdar JA, 2014. Assessment of bacteriological quality of ready to eat food vended in streets of Silchar city, Assam, India. Indian J Med Microbiol 32: 169-171.

41. Lumsden LL, 1917. The determination of ice cream as a factor in the spread of typhoid fever infection. Am J Public Health (N Y) 7: 1005-1009.

42. Taylor A Jr., Santiago A, Gonzalez-Cortes A, Gangarosa EJ, 1974. Outbreak of typhoid fever in Trinidad in 1971 traced to a commercial ice cream product. Am J Epidemiol 100: 150-157.
43. Tauxe RV, 2002. Emerging foodborne pathogens. Int J Food Microbiol 78: 31-41.

44. Mitscherlich E, 1984. Microbial Survival in the Environment. New York, NY: Springer-Verlag.

45. Masud T, 1989. Microbiological quality and public health significance of ice-cream. J Pak Med Assoc 39: 102-104.

46. Hancock-Allen J, Cronquist AB, Peden J, Adamson D, Corral N, Brown K, 2016. Notes from the field: typhoid fever outbreak associated with an asymptomatic carrier at a restaurant-weld county, Colorado, 2015. MMWR Morb Mortal Wkly Rep 65: 606-607.

47. Olsen SJ, Bleasdale SC, Magnano AR, Landrigan C, Holland BH, Tauxe RV, Mintz ED, Luby S, 2003. Outbreaks of typhoid fever in the United States, 1960-99. Epidemiol Infect 130: 13-21.

48. Lynch MF et al., 2009. Typhoid fever in the United States, 1999-2006. JAMA 302: 859-865.

49. Lin FY, Becke JM, Groves C, Lim BP, Israel E, Becker EF, Helfrich RM, Swetter DS, Cramton T, Robbins JB, 1988. Restaurantassociated outbreak of typhoid fever in Maryland: identification of carrier facilitated by measurement of serum $\mathrm{Vi}$ antibodies. J Clin Microbiol 26: 1194-1197.

50. Ali M, Emch M, Donnay JP, Yunus M, Sack RB, 2002. Identifying environmental risk factors for endemic cholera: a raster GIS approach. Health Place 8: 201-210.

51. Sasaki S, Suzuki H, Igarashi K, Tambatamba B, Mulenga P, 2008. Spatial analysis of risk factor of cholera outbreak for 2003-2004 in a peri-urban area of Lusaka, Zambia. Am J Trop Med Hyg 79: 414-421.

52. Waddington CS et al., 2014. An outpatient, ambulant-design, controlled human infection model using escalating doses of Salmonella Typhi challenge delivered in sodium bicarbonate solution. Clin Infect Dis 58: 1230-1240.

53. Hornick RB, Greisman SE, Woodward TE, DuPont HL, Dawkins AT, Snyder MJ, 1970. Typhoid fever: pathogenesis and immunologic control. N Engl J Med 283: 686-691. 